Acta Crystallographica Section A

Foundations of Crystallography

ISSN 0108-7673

Received 3 July 2007

Accepted 6 August 2007

C) 2007 International Union of Crystallography

Printed in Singapore - all rights reserved

\section{Three-periodic nets and tilings: natural tilings for nets}

\author{
Vladislav A. Blatov, ${ }^{a}$ Olaf Delgado-Friedrichs, ${ }^{\text {b,c }}$ Michael $\mathrm{O}^{\prime}$ Keeffe $^{\mathrm{b} *}$ and \\ Davide M. Proserpio ${ }^{\mathrm{d}}$
}

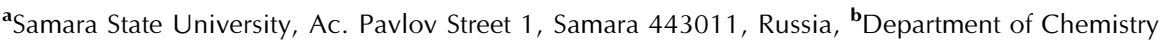
and Biochemistry, Arizona State University, Tempe, AZ 85287, USA, 'Supercomputer Facility, The Australian National University, Canberra, ACT 2600, Australia, and d Dipartimento di Chimica Strutturale e Stereochimica Inorganica (DCSSI), Università di Milano, Via G. Venezian 21, 20133 Milano, Italy. Correspondence e-mail: mokeeffe@asu.edu
\end{abstract}

Rules for determining a unique natural tiling that carries a given three-periodic net as its 1-skeleton are presented and justified. A computer implementation of the rules and their application to tilings for zeolite nets and for the nets of the RCSR database are described.

\section{Introduction}

Chemists have long had an interest in describing the topology of crystal structures in terms of nets (Wells, 1977, 1979). Recently, there has been substantial interest in systematic enumeration of periodic nets (Hyde et al., 2006; Treacy et al., 1997, 2004; Blatov, 2007) and one of the most fruitful methods has been the enumeration of periodic tilings, and hence the nets they carry (Delgado-Friedrichs et al., 1999; DelgadoFriedrichs \& Huson, 2000; Delgado-Friedrichs \& O'Keeffe, $2005 a, 2006,2007)$. At the same time, it has proved fruitful to discuss tilings associated with a particular net and to use them in a classification scheme for nets (Delgado-Friedrichs et al., $2003 a, b, 2006,2007)$. We indicate later other reasons for associating a tiling with a net. However, although a tiling carries a unique net of edges and vertices, for a given net there may be many possible tilings that carry that net, or perhaps none at all. However, we have developed the idea of a unique natural tiling (Delgado-Friedrichs et al., 2003a), although we acknowledge the earlier related work of Schoen (1970). For some complicated nets that arise in crystal chemistry and elsewhere, the rules given earlier have to be elaborated to result in a unique tiling. These are the subject of this paper. We start with some informal definitions.

\section{Definitions}

A net is a special kind of graph. It is simple - the edges are undirected and there are no multiple edges or loops, and it is connected - there is at least one path between every pair of vertices. All the nets discussed in this paper are three-periodic, i.e. their automorphism groups contain translations in three non-coplanar directions. For a more complete list of terms and definitions relevant to the consideration of crystal nets as graphs, see Delgado-Friedrichs \& O'Keeffe (2005b).

Tilings divide Euclidean space into tiles and again all the tilings we discuss are three-periodic. They are always face-to- face, i.e. a face of a tile is shared by exactly two tiles. A tile is the interior of a generalized polyhedron (a cage) that may contain divalent vertices and is topologically equivalent to a sphere.

The edges and vertices of a polyhedron sensu stricto form a net that is a planar three-connected graph. A simple polyhedron has exactly three faces and three edges meeting at each vertex. A simple tiling is a tiling by simple polyhedra in which four tiles meet at each vertex, three at each edge and two at each face. Foams are simple tilings by bubbles.

A tiling is proper if the automorphism group of the tiling is the same as that of the graph it carries (its 1-skeleton). The nets we consider here in the context of natural tilings are crystallographic in the sense that they have an automorphism group that is isomorphic to a three-dimensional space group and it is this symmetry that is implied when we talk about the symmetry of a net unless we explicitly refer to a lowersymmetry embedding. For a discussion of nets for which the automorphism group may not be isomorphic to a space group - e.g. some nets with 'collisions' in a barycentric placement see Delgado-Friedrichs \& O'Keeffe (2003). As a periodic tiling always has a space-group symmetry, non-crystallographic nets do not have a proper tiling as defined above.

The dual of a tiling is a second tiling obtained as follows. A new vertex is placed inside each original tile and connected to the new vertices in adjacent tiles sharing a common face by an edge through that face. To complete the construction, new tiles are constructed so that the dual of the dual is the original tiling. If a tiling and its dual are identical, the tiling is self-dual. The dual of a proper tiling need not be, and often is not, a proper tiling; an example is shown in Fig. 10 below.

The transitivity of a tiling is a sequence of four integers pqrs that indicates that the tiling has $p$ types of vertex, $q$ types of edge, $r$ types of face and $s$ types of tile.

The vertices and edges of the faces of tiles are polygons, not necessarily planar. In the language of graph theory, they are cycles of the graph. Special kinds of cycles are rings, which are 
cycles that are not the sum of two smaller cycles, and strong rings, which are cycles that are not the sum of any number of smaller cycles (Goetzke \& Klein, 1991; see also DelgadoFriedrichs \& O'Keeffe, 2005b). A ring that is not a strong ring is a weak ring.

It is sometimes useful to give face symbols for tiles. These are of the form $\left[M^{m} . N^{n} \ldots\right]$ and indicate that there are $m$ faces that are $M$-rings, $n$ faces that are $N$-rings etc. Conventionally, $M<N<\ldots$ The set of face symbols for a tiling, e.g. $2\left[3^{4}\right]+\left[3^{8}\right]$ for a space filling by tetrahedra and octahedra in the ratio $2: 1$ is called the signature of the tiling.

Nets are given a symbol consisting of either three letters in boldface as in abc or three letters and extensions as in abc-d or abc-d-e. Crystallographic and other data can be found for these nets in the RCSR database at http://rcsr.anu.edu.au/.

\section{Some nets with unique proper tilings}

The simplest, and most regular, tiling of Euclidean space is the space-filling by equal cubes, Fig. 1. The symmetry of the tiling and the net (symbol pcu) is $P m \overline{3} m$. It should be clear that we could make a tiling using double cubes (cubes sharing a face, Fig. 1) or indeed multiple cubes in a great variety of ways - in fact, an infinite number of ways. We could also divide the cube into smaller units as also shown in Fig. 1. Indeed, a cube can be subdivided in 12 distinct ways without introducing new vertices or edges (Bonneau et al., 2004) and these subdivided cubes can be again assembled into many distinct tilings of pcu. One can also subdivide the fused cubes. However, it is easy to

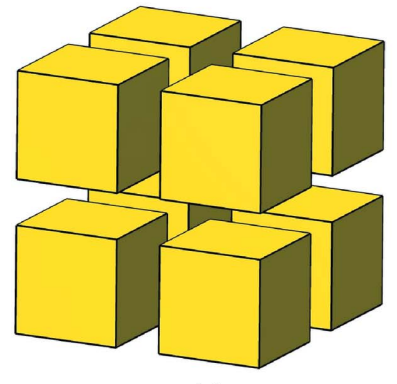

(a)

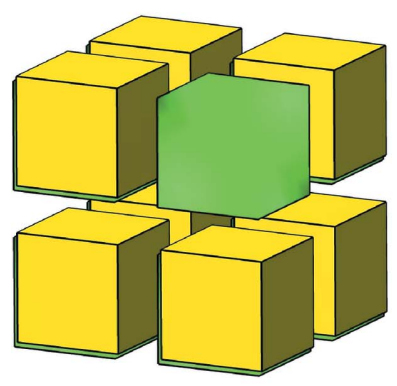

(c)

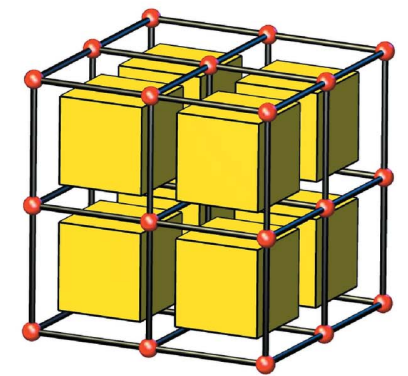

(b)

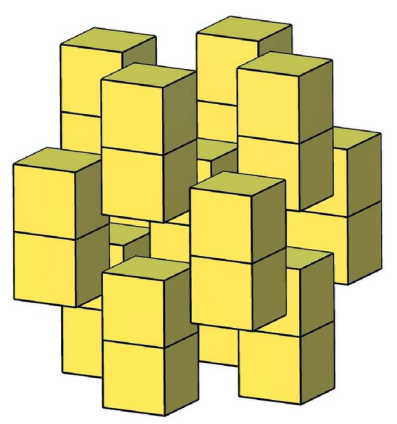

(d)
Figure 1

(a) A tiling by cubes with the tiles slightly shrunk for clarity. (b) The same with edges and vertices of the net emphasized. (c) Part of a tiling by half cubes. (d) Part of a tiling by double cubes. show that all these additional tilings will have lower symmetry so there is only one proper tiling of pcu. Specifically, all subdivisions of a cube destroy some of the symmetries of the cube, and all tilings by multiple cubes must lose some translations. So in this case the proper tiling is unique and we call it a natural tiling. Our first rule for a natural tiling is then $(a)$ the symmetry of the tiling must be the same as that of the net.

Examples of other nets for which the proper tiling is unique because of the high symmetry are the nets of $\mathrm{Si}$ in $\mathrm{SrSi}_{2}$ ( $\mathbf{s r s}$ ), of $\mathrm{NbO}$ (nbo), diamond (dia) and the nets of the bodycentered cubic (bcu) and face-centered (fcu) lattices (These are the regular and quasiregular nets of Delgado-Friedrichs et al., 2003a). It turns out that examination of approximately 1400 different nets in the RCSR database showed that over 500 have a unique proper tiling, but most of the rest admit more than one such tiling (for a small fraction, we have found no proper tiling). Indeed, a given net may admit some hundreds of proper tilings (we give an example later). Our search for natural tilings for these nets is guided in part by the desire to preserve the tiles found in unique proper tilings when they occur.

\section{Further rules for natural tilings}

Fig. 2 shows a tile of bcu and illustrates that the structure contains two kinds of 4-ring, necessarily strong rings as they are the smallest cycles in the structure. One set (non-planar, see Fig. 2) forms faces of the tiles. The second set (planar) intersect (cross) so that not all can be faces of tiles. Thus the net can have a tiling in which the 4-face tile (a tetrahedron) is subdivided into 3-face tiles (trihedra) but this can only use one half of the planar rings reducing the symmetry from cubic to at

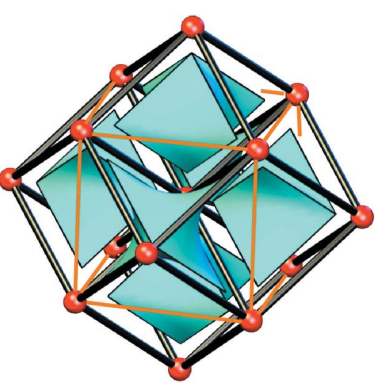

(a)

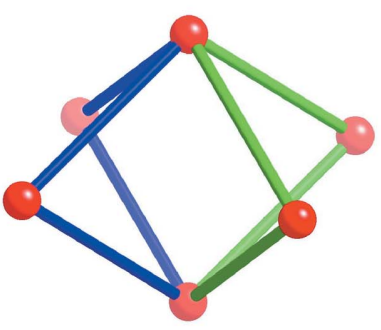

(c)

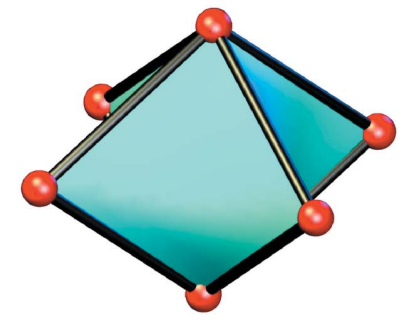

(b)

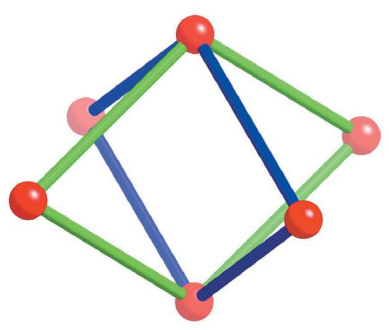

(d)
Figure 2

(a) Tiling for the net of the body-centered cubic lattice; tiles shrunk for clarity. (b) One tile. (c) The skeleton of one tile with two face rings shown as blue and green respectively. $(d)$ The same skeleton but now blue and green outline a pair of crossing rings. 
most tetragonal. This example shows that to make a proper tiling we must exclude as candidates for tiles rings that have crossings with other rings of the same kind.

The detection of crossing is not as simple as this example suggests. Ideally we would like a purely topological criterion as nets and tilings are primarily topological constructs with embeddings being of only secondary importance (but, of course, vital in crystal-chemical applications). Clearly, we must consider the whole structure - not just the tile. Indeed, considering the skeleton of the bcu tile as a graph, the six rings are all equivalent. In practice, at present we work with an embedding. There is only one embedding of bcu with full symmetry $(\operatorname{Im} \overline{3} m)$ and it is 'obvious' which rings cross and which do not. But to show that the problem is non-trivial, we show (Fig. 3) a tile from another net, ifi, which again has vertices in fixed positions at maximum symmetry $\left(I 4_{1} 32\right)$. The faces are all very non-planar and it is not trivial to find an algorithm that decides whether the rings intersect. Indeed, in the paper introducing this net (Delgado-Friedrichs et al., 2006), no tiling was given.
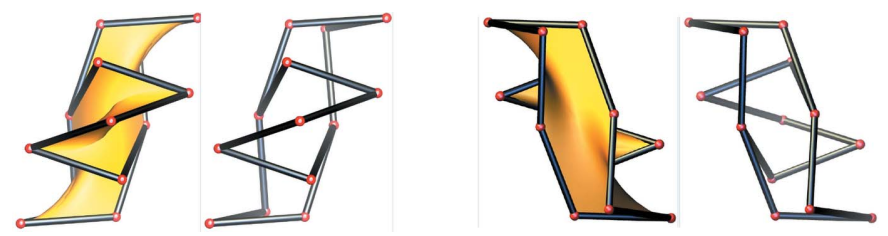

Figure 3

Views from two directions of one of the $\left[8.10^{2}\right]$ tiles for the net ifi tile exhibiting very twisted 10 -sided faces.
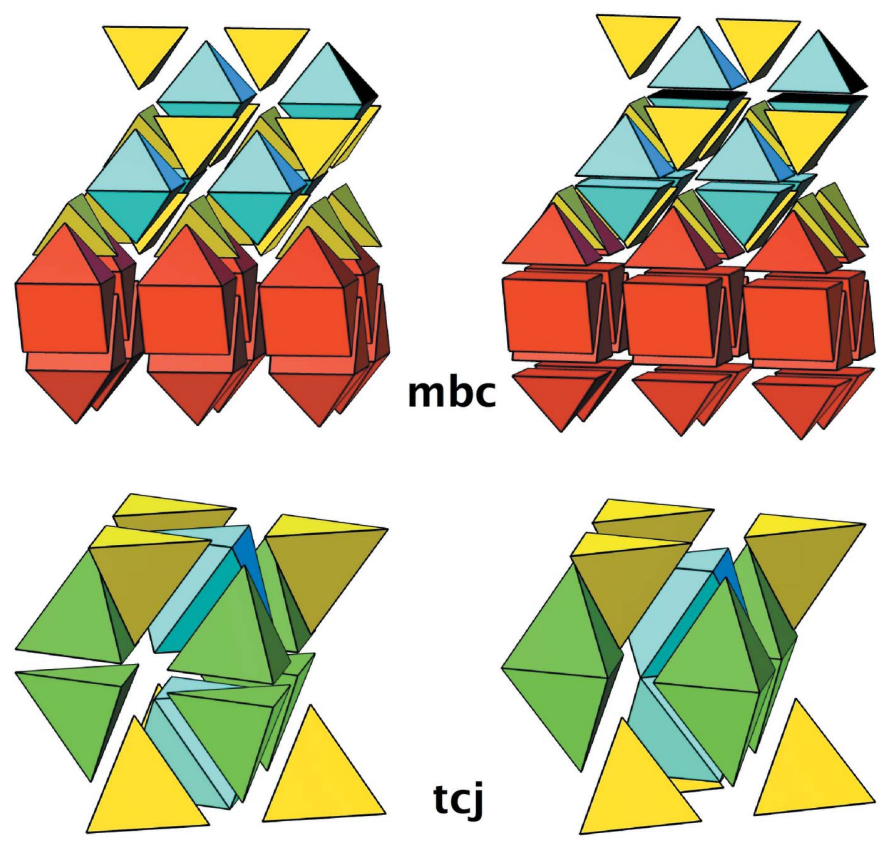

Figure 4

Top: tilings for mbc: left a natural tiling $3\left[3^{4}\right]+\left[3^{8}\right]+\left[3^{6} \cdot 4^{2}\right]$ (yellow, cyan, red), right a tiling $3\left[3^{4}\right]+3\left[3^{4} \cdot 4\right]+\left[3^{2} \cdot 4^{3}\right]$ violating rule $(b)$. Bottom: tilings for tcj: left a natural tiling $\left[3^{4}\right]+\left[3^{4}\right]+\left[3^{8}\right]$ (yellow, green, cyan), right a tiling $2\left[3^{4}\right]+\left[3^{6}\right]+2\left[3^{8}\right]$ violating rule $(c)$.
Examination of the tiles for nets that have unique proper tilings suggests the following further rules for constructing natural tiles when there is more than one possible proper tiling for a net. (b) We require that the tiles do not have one face that is larger (has more edges) than the other faces of the tile; as discussed below ( $\S 6)$, we call this the condition that the faces are locally strong (in what follows 'strong' means 'locally strong'). In $\S 8$, we discuss a rarely occurring situation in which we may want to override this rule. (c) If a tile has non-face strong rings and if further these rings do not intersect other such rings, the tile is split so that these rings become faces of smaller tiles.

The reasons for these rules are so that tiles such as the tetrahedra and octahedra of the net (fcu) of the face-centered cubic lattice are conserved in tilings of lower-symmetry nets. Rule $(b)$ ensures that even when allowed by symmetry a tile such as an octahedron is not subdivided into e.g. two square pyramids. Similarly, rule $(c)$ ensures that tiles such as a fused pair of face-sharing tetrahedra or octahedra are divided into pairs of tetrahedra or octahedra, respectively. Examples are shown in Fig. 4. There the net with symbol mbc (this is the Mo structure in $\mathrm{Mo}_{2} \mathrm{BC}$ ) contains octahedra which can be dissected into pairs of square pyramids without lowering the symmetry $(\mathrm{Cmcm})$ but forbidden by rule $(b)$. In the net $\mathbf{t c j}$ (this is the net of $h c=A B A C$ closest sphere packing), the symmetry $\left(P 6_{3} / m m c\right)$ allows the fusion of pairs of tetrahedra or pairs of octahedra, but rule $(c)$ forbids this.

These rules are still insufficient to yield a unique natural tiling for more-complex nets. There remains the case where tiles contain intersecting non-face strong rings. Then we use rule $(d)$ : if one of a pair of intersecting strong rings is smaller
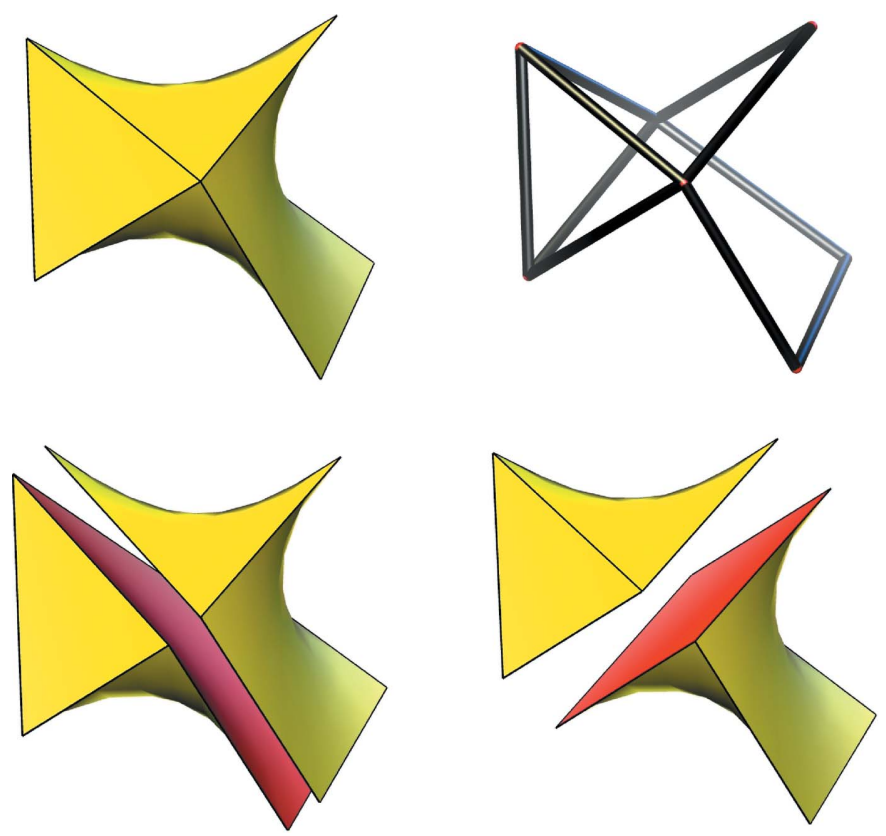

Figure 5

Top: a tile $\left[3^{2} .4 .5^{2}\right]$ of an isohedral tiling of the net eci with its skeleton on the right. Bottom: two tilings $\left[4.5^{2}\right]+\left[3^{2} .5^{2}\right]$ (left) and $\left[4.5^{2}\right]+\left[3^{2} .4^{2}\right]$ (right) compatible with rules $(a)-(c)$ derived by splitting that tile. Rule $(d)$ selects the one on the right as the natural tiling. 
than the other, the larger is rejected as a possible face. If the intersecting rings are the same size, we reject both as possible faces.

We now give examples of application of rule $(d)$. The net eci has an isohedral tiling (Fig. 5) $\left[3^{2} .4 .5^{2}\right]$ that contains intersecting non-face strong 4-rings and 5-rings. Using the 4-ring to split the tile gives the tiling $\left[3^{2} \cdot 4^{2}\right]+\left[4.5^{2}\right]$ and splitting using the 5-ring gives the tiling $\left[3^{2} \cdot 5^{2}\right]+\left[4.5^{2}\right]$. Both the last two obey rules $(a)$ to $(c)$ but we prefer the former as the natural tiling because one of the tiles has smaller faces.

The net with symbol mef with symmetry $\mathrm{Cmmm}$ has two different tilings $\left[6^{3}\right]+\left[6^{5}\right]$ (Fig. 6). We note however that the two possibilities correspond to two different dissections of a tile $\left[6^{4}\right]$ into two tiles $\left[6^{3}\right]$. We note further that the $\left[6^{4}\right]$ tile occurs in other higher-symmetry nets such as mot (symmetry $P 4 / \mathrm{mmm}$ ), where it cannot be split without lowering the symmetry (mot is an example of a net with a unique proper tiling). As we want the same tiles to be part of natural tilings of different nets ( $c f$. the discussion of tetrahedra and octahedra above), we do not use either of the intersecting 6-rings as faces and the natural tiling is $\left[6^{4}\right]+2\left[6^{5}\right]$ (Fig. 6 ). To further justify this procedure, we recall that intersecting strong 4-rings are rejected as possible faces in the tiling of bcu.

\section{Two-face edges and pairs of tiles sharing more than one face}

We would prefer that at least three tiles meet at an edge. If only two faces meet at an edge, those two faces must be shared by the same pair of tiles. Accordingly, the dual structure will have a pair of vertices joined by two edges. So, two-face edges are to be avoided if possible; this is usually provided by the rules $(a)-(d)$. However, sometimes two-face edges cannot be
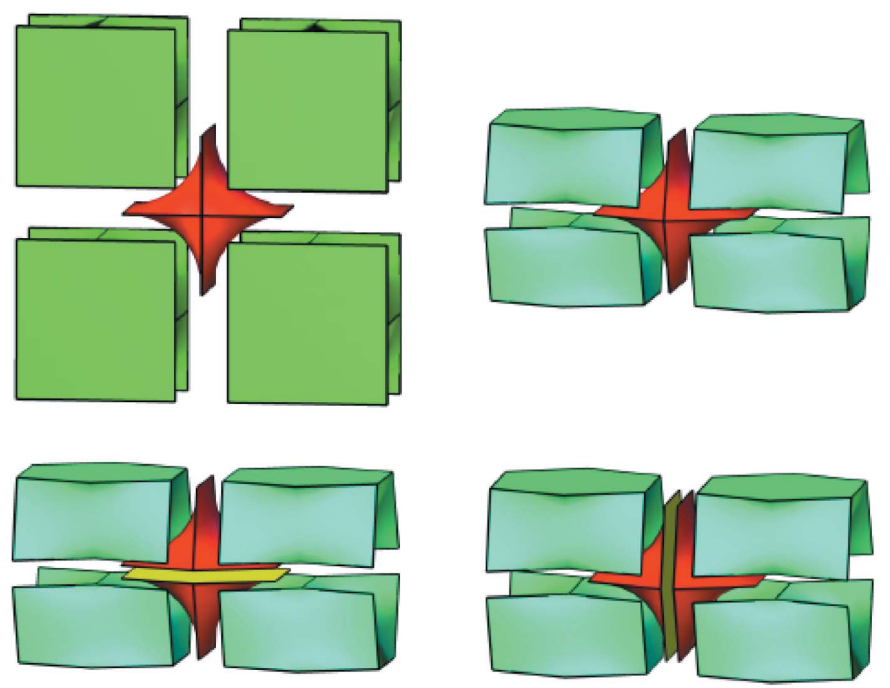

Figure 6

Top left: natural tiling $\left[6^{4}\right]+\left[6^{4} .8^{2}\right]$ for net mot. The other three tilings (from top right clockwise: $\left[6^{4}\right]+2\left[6^{5}\right]-$ red and green respectively; $\left[6^{3}\right]+$ $\left.\left[6^{5}\right] ;\left[6^{3}\right]+\left[6^{5}\right]\right)$ are for mef and all obey rules $(a),(b)$ and $(c)$. The one on the top right $\left[6^{4}\right]+2\left[6^{5}\right]$ obeys rule $(d)$ as well and has the same tile (red) as mot. avoided - Fig. 7(a) gives a simple example of a tiling by tiles that are topologically equivalent to pentagonal dodecahedra $\left[5^{12}\right]$. From the point of view of the tiling, the two-face edges are superfluous and one could remove them; however, from the point of view of the net (cdh), all edges are an integral part of the structure and their removal would simply give another net. In this example, one must either accept the tiling shown or concede that there is no natural tiling. We prefer the first alternative as we have found that several zeolite nets only admit tilings with two-face edges.

Another simple example (tiling of net fsh) is shown in Fig. $7(b)$. In this case, there is an alternative tiling without two-face edges as shown in Fig. 7(c). However, in this last case, there are tiles [yellow in Fig. $7(c)]$ that are $\left[4^{3} .8\right]$ and thus violate rule $(b)$ by having one face larger than all the rest, so we prefer the first alternative (Fig. 7b).

As a variation on this theme, we call attention to the tiling for the zeolite framework $\mathbf{U O Z}$ shown in Fig. 8. Pairs of blue tiles share two hexagonal faces so the dual has the same problem of vertices linked by multiple edges. Notice that this situation cannot be avoided without lowering the symmetry of the tiling. However, in this case, there are no two-face edges. In fact, four tiles meet at each vertex, three at each edge and two at each face, but the tilings are not simple because the tiles are not all simple polyhedra.

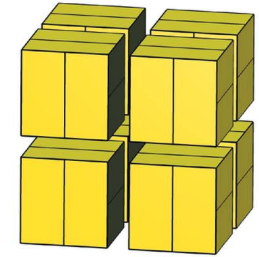

(a)

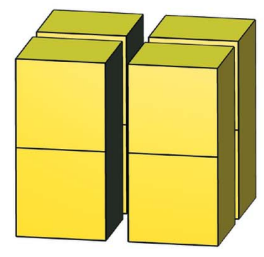

(b)

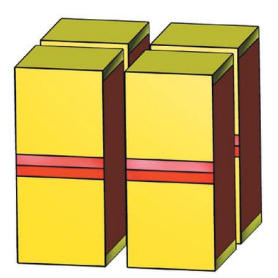

(c)
Figure 7

(a) A tiling by dodecahedra, $\left[5^{12}\right]$ (net $\mathbf{c d h}$ ); the long edges are two-face edges. (b) Another tiling with two-face edges (net fsh) with $\left[4^{6} .6^{2}\right]$ tiles. (c) An alternative non-natural tiling for the net in $(b)$ with red tiles $\left[6^{2} .8^{2}\right]$ and yellow tiles $\left[4^{3} .8\right]$, the latter have a weak-ring face.

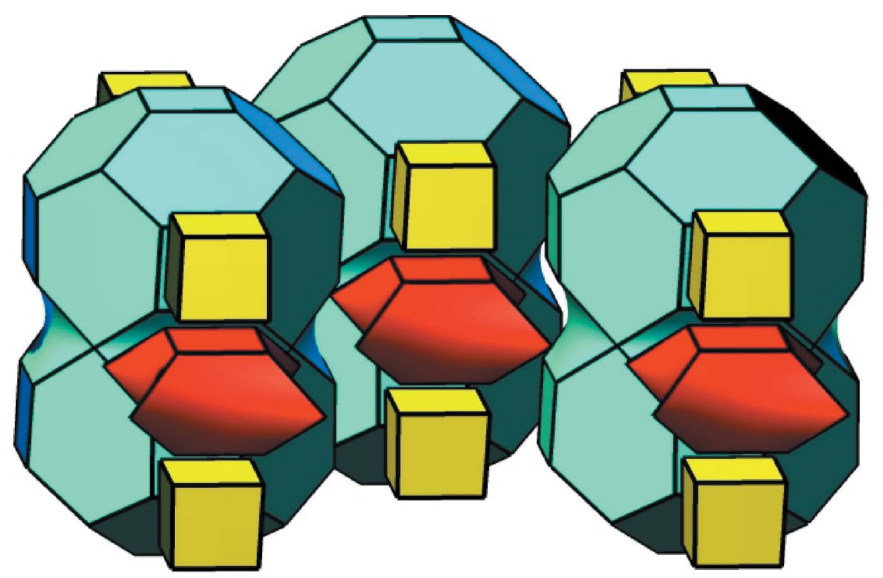

Figure 8

Part of a tiling $2\left[4^{6}\right]+\left[4^{2} .6^{4}\right]+\left[4^{10} .6^{20}\right]$ for the net of the zeolite framework UOZ. Pairs of blue tiles share two hexagonal faces. 


\section{Locally or globally strong rings?}

Rule $(b)$ requiring that no face of the tile is larger than all the rest is automatically satisfied if only strong rings (rings that are not the sum of smaller rings) are allowed. However, there are practical problems in finding strong rings in a complicated structure. We know that there is a finite number of rings (and hence strong rings) but we do not know a priori how large the largest ring is, and, as there is an infinite number of cycles of ever increasing size, the problem of knowing when to stop examining cycles to see if they are rings is not trivial. Even if we have a complete set of rings, the problem of determining whether a given ring is strong can require examination of sums involving rings far from the ring in question. Fig. 9 illustrates this last point. It shows part of a tiling by three kinds of tile; the red tile has face symbol $\left[3^{18} \cdot 6^{7}\right]$ so no one face is bigger than the rest. However, as shown in the figure, groups of tiles can be joined together to give a solid (not a tile because there are internal edges and faces) with face symbol $\left[3^{6} .4^{18} .6\right]$ so that the 6-ring (the only one visible in the right part of the figure) is the sum of six 3-rings and eighteen 4-rings and thus not a strong ring. Nevertheless, we accept that ring as the face of a tile in a natural tiling.

In the context of molecular chemistry, strong rings are called relevant cycles (Berger et al., 2004). However, in view of the difficulties alluded to above, we prefer to consider the faces of the tiles of a natural tiling as a uniquely defined fundamental set (we believe that all rings of the net can be expressed as a sum of these) which we call essential rings (Delgado-Friedrichs et al., 2003a). The essential rings are not necessarily a minimal set, however. Indeed, for some nets we find there are several maximal proper tilings (tilings with minimal transitivity) and we have not developed criteria for preferring one of these over the others.
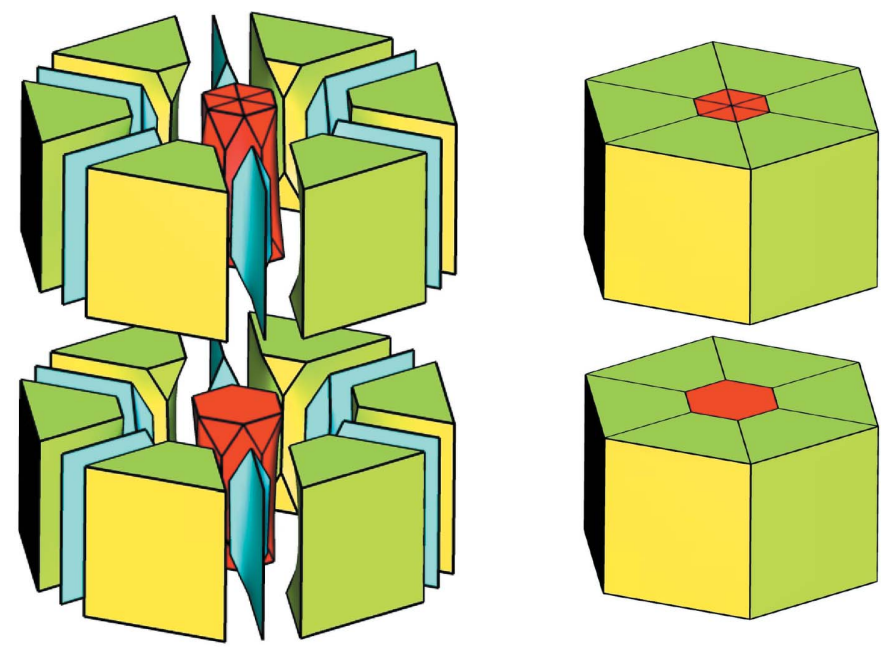

Figure 9

Part of a tiling with three kinds of tile: $\left[6^{3}\right]$ (blue), $\left[3^{2} \cdot 4^{2} \cdot 6^{2}\right]$ (green) and $\left[3^{18} \cdot 6^{7}\right]$ (red). No ring is locally weak, but as shown on the right one 6-ring is globally weak.

\section{Nets with catenated rings}

It has long been recognized that the nets of some known crystals such as the coesite form of $\mathrm{SiO}_{2}$ have rings that are catenated by other rings (O'Keeffe, 1991) and many more examples have been found in metal organic frameworks (Carlucci et al., 2003a,b). Clearly such rings are not eligible to be faces of tiles, but tilings still may exist for the net, as indeed is the case for the coesite net (coe). Elsewhere (DelgadoFriedrichs et al. 2005), attention was drawn to a net fnu that can be derived as follows. Take two diamond (dia) nets that are the nets of a pair of self-dual dia tilings. Every 6-ring of one net is catenated with a 6-ring of the dual net. Now to form fnu make a link between pairs of vertices on separate nets so that every vertex is now 5-coordinated. The additional link makes additional 6-rings and it transpires that these alone are sufficient to make a tiling for the new net as shown in Fig. 10 and this tiling is a unique natural tiling for the net.

Also shown in the figure is the tiling dual to that for fnu. It may be seen that the tiling is not natural as a $\left[6^{8}\right]$ tile has been split into two [6 $\left.6^{4} .12\right]$ tiles. The net of the dual tiling is in fact that of the NbO net (nbo) for which the $\left[6^{8}\right]$ tiling is natural.

\section{Some remaining problems}

Nets without full-symmetry faithful embeddings. As has been discussed elsewhere (Delgado-Friedrichs \& O'Keeffe, 2003; Delgado-Friedrichs et al., 2005), for a small class of nets there

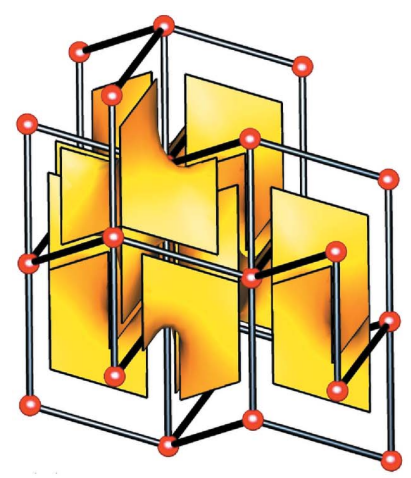

(a)

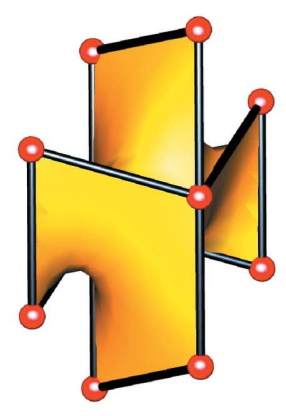

(c)

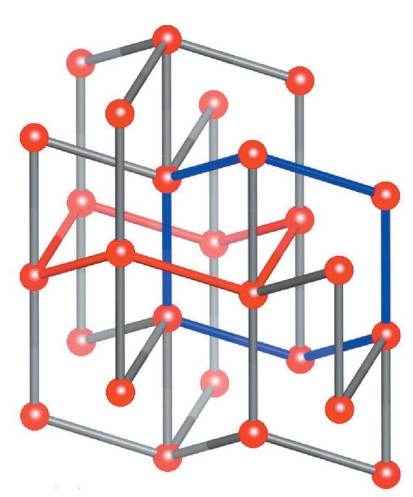

(b)

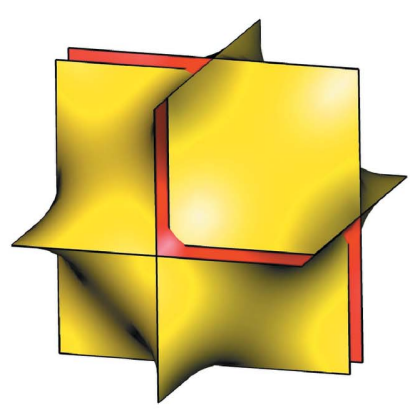

(d)
Figure 10

(a) Part of a tiling for the net fnu. (b) The net showing two catenated rings (blue and red). (c) One tile $\left[6^{4}\right]$. (d) Two tiles of the dual tiling which carries net nbo. 
is not a faithful embedding at maximum symmetry. By this we mean that, in the maximum symmetry, edges may of necessity intersect at points that are not vertices. A common reason is that, at full symmetry, a $\left[3^{4}\right]$ tetrahedron collapses to a square with intersecting diagonals on a mirror plane, so a tile (a tetrahedron is necessarily a natural tile) collapses to zero volume. Although rare, it is not unknown in crystal chemistry (see the example of the net of moganite anions, discussed by Delgado-Friedrichs et al., 2005) and we have to do what nature does and use a lower-symmetry embedding.

Non-crystallographic nets. There are nets with automorphisms that do not correspond to crystallographic symmetry operations (Delgado-Friedrichs \& O'Keeffe, 2003). We can still find tilings for them - see for example the net bcr discussed by Delgado-Friedrichs et al. (2005). As they have yet to be encountered in crystal chemistry, we defer further consideration until another occasion.

Possible tiling with tile faces that are weak rings. In a survey (unpublished data) of natural tilings for zeolite nets, we came across a few examples where we felt it might be more 'natural' to split larger tiles into smaller components, some of which have weak rings as faces. Two examples are shown in Fig. 11.

To find a possible criterion for when to split, we note that, for a tiling of a two-dimensional surface of genus $g$ with $k$ vertices, each with vertex symbol $n_{1} \cdot n_{2} \ldots n_{i}$, Euler's equation may be written (cf. O'Keeffe \& Hyde, 1996, p. 407)

$$
\sum_{k} \delta_{k}=\sum_{k}\left[1-\sum_{i}\left(1 / 2-1 / n_{i}\right)\right]=2-2 g
$$

$2 \pi \delta_{k}$ is the angular deficit, so called because if the polygons $n_{i}$ meeting at the vertex were regular its value would be the difference $2 \pi-$ sum of the angles at the $i n$-gons. For a closed surface (polyhedron) with $g=0$, the sum is positive; for plane, torus etc., with $g=1$, the sum is zero; for surfaces with higher genus (negative curvature), the sum is negative. We propose that, if a tile has one or more 'waists' that are weak rings, the tiles can be split at that ring if $(a) \delta$ for all the vertices on that ring is negative, and $(b)$ all the vertices on that ring have valence four or greater. According to these criteria, the tiles in Fig. 11 are split as shown. In these examples, the larger tile after the split is the $\alpha$-cage, which occurs in the nets of several zeolites, for example LTA and RHO in which the $\alpha$-cage is part of the unique proper tiling. We agree that the rule is arbitrary but, in our experience, the need for it is rare (e.g. for only 4 of 176 zeolite nets) and occurs only for relatively complex nets. Indeed, rather than considering it a rule, we should think of it as a dispensation to break rule $(b)$ in exceptional circumstances. When it is applied, we can signify the fact by referring to the tiling as a 'modified natural tiling'.

\section{What use are tilings for nets?}

The reader at this point may be wondering what are the advantages of having a tiling for a net. Here we list some that occur to us.

(a) The natural tiling provides a natural division of space by the net that enables the size and location of the cavities

('holes') to be identified. This is particularly satisfying if the number of kinds of tile is small. In fact, the natural tiling gives all 'topological' cavities in the net, i.e. the cavities irrespective of their size and shape. For a particular embedding of the net, we may then decide what cavities are significant from the geometrical point of view, for instance, to include interstitial particles of a given size.

(b) The faces of the tiles provide a complete set of rings in the structure (essential rings) in the sense that all other rings are combinations of these. Like the correspondence between tiles and cavities, they represent a set of windows or channels connecting cavities.

(c) The occurrence of the same tiles, or sets of tiles, in separate structures suggests structural relationships.

(d) It is possible that in some cases particular tiles may form in the synthetic process due to structure-directing agents such as templates. The recognition of tiles in hypothetical structures may then lead to identification of possible synthetic strategies.

(e) Associated with the tiling is a transitivity. This is very useful in establishing a taxonomy of nets (Delgado-Friedrichs et al., 2007).

$(f)$ Every tiling has a dual, so for every net we can identify a second net ('dual net') that is the net of the dual of the natural tiling. Nets with self-dual tilings tend to occur in crystals as intergrown/interpenetrated pairs or multiplets [for more on

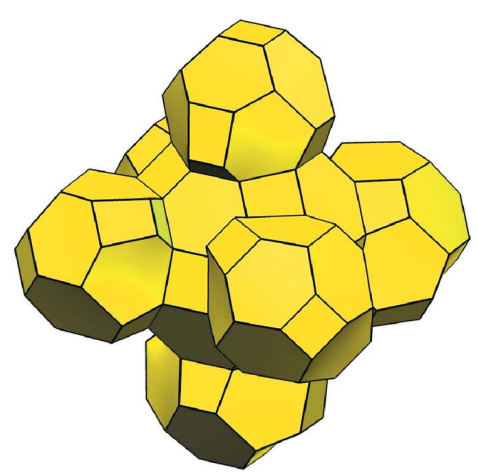

LTN

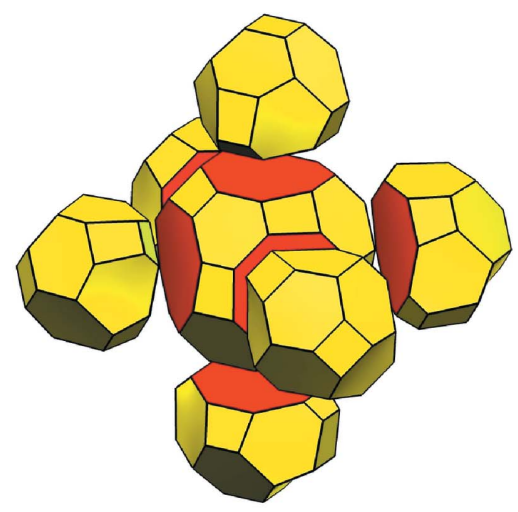

Figure 11

Tiles of tilings for nets of zeolite frameworks LTN and UFI. Top: single tiles if no weak-ring faces are allowed. Bottom: The same split by weak rings $\left(\right.$ red). LTN $\left[4^{54} \cdot 6^{56}\right] \rightarrow 6\left[4^{7} \cdot 6^{8} \cdot 8\right]+\left[4^{12} \cdot 6^{8} \cdot 8^{6}\right]$, UFI $\left[4^{22} \cdot 5^{8} \cdot 6^{16} \cdot 8^{4}\right] \rightarrow$ $2\left[4^{5} \cdot 5^{4} \cdot 6^{4} \cdot 8\right]+\left[4^{12} \cdot 6^{8} \cdot 8^{6}\right]$. 
interpenetration, see Blatov et al. (2004) and Baburin et al. (2005)]. The dual net corresponds to the system of cavity centers and channel lines and can represent, for instance, the conduction pattern in fast-ion conductors (cf. Blatov et al., 2006).

$(g)$ The tiles of the dual net are, in a way, the space 'belonging' to the original vertex in that tile - what Schoen (1970) refers to as the domain of a vertex. This idea leads to a nice description of the structures of complex intermetallic compounds (O'Keeffe, to be published).

\section{Summary of rules, their computer implementation, and results}

The rules that we propose for determining a unique natural tiling are

(a) the tiling must have the symmetry of the net (be proper);

(b) the faces of tiles must be locally strong rings (no tile has one face larger than the rest);

(c) a tile does not have non-face strong rings that do not intersect other such rings;

(d) when rules $(a)-(c)$ result in multiple tilings because of intersecting strong rings, we use only the smaller of a pair if the intersecting rings are unequal in size and reject both if the intersecting rings are equal in size;

(e) rule (b) may be overridden for tiles with waists of all negative-curvature vertices.

The program TOPOS (Blatov, 2006, see also http:// www.topos.ssu.samara.ru/) has among its many capabilities the ability to determine natural tilings. The following algorithm is used. (i) All rings up to a certain size are found. In practice, all rings can almost always be found by making this size sufficiently large. (ii) Rings are then typified as weak or strong by checking all ring sums up to a specified sum size $\left(n_{\max }\right)$ and weak rings rejected. (iii) Further rings are then rejected as not forming an essential set of faces if they are catenated with other rings or if they intersect with other rings of the same kind. (iv) The remaining rings are arranged into sets such that no pair of rings in a set intersect (intersecting rings cannot both be tile faces). (v) Candidate tilings are formed from each of these sets of strong rings. (vi) If there are tiles with locally weak rings, $n_{\max }$ is increased up to $n_{\mathrm{f}}-1$, where $n_{\mathrm{f}}$ is the number of faces in the largest tile of this type and the procedure repeats starting from step (ii). (vii) If there is more than one possible tiling, then a tiling is formed rejecting intersecting rings unless one is smaller than the others [this is rule $(d)$ ]. (viii) The conditions described in $§ 8$ may optionally be used to find 'waists' of the tiles and to get a modified natural tiling.

For complicated nets, these calculations are far from trivial and would be impossible to do by hand. Let us consider the net of the zeolite framework USI. At the first step, TOPOS finds 26 kinds of non-equivalent ring on checking all circuits up to size 20: five 4-rings, eight 6-rings, one 10-ring, six 12-rings and six 16-rings. Applying rule (a) only, it is found that the net admits 752 different proper tilings utilizing different sets of these rings.
However, one 6-ring is included in tiles [ $\left.4^{3} .6\right]$, so it is the sum of three smaller rings and hence a weak ring. Using $n_{\max }=$ 3, TOPOS rejects it at the second step.

At step (iii), TOPOS determines that there are no catenated rings in the net, but one 6-ring, three 12-rings (12a, 12b, $12 c$ ) and all 16-rings cross with other rings of the same kind and are also to be rejected as inessential rings (they cannot be faces in proper tilings).

At the next step, TOPOS finds that two remaining 12-rings (12e and 12f) cross with each other and therefore cannot belong to the same set of essential rings. Thus there are two sets of essential rings that form two tilings at step (v) according to rules $(a)-(c)$. These tilings are similar and have the same signature $4\left[6^{3}\right]+2\left[4^{2} \cdot 6^{2}\right]+\left[4^{2} \cdot 6^{4} \cdot 10^{2}\right]+2\left[4^{6} \cdot 6 \cdot 12^{2}\right]+$ $\left[4^{8} \cdot 6^{2} \cdot 10^{2} \cdot 12^{2}\right]$, but the tiles $\left[4^{6} \cdot 6 \cdot 12^{2}\right]$ in different tilings contain different 12-rings: $12 e$ or $12 f$. There are no more locally weak rings in those tilings [step (vi)], so rule $(d)$ may be applied and both the $12 e$ and $12 f$ rings rejected. Accordingly, at the last step [(vii)], TOPOS uses all 4-rings, six 6-rings (except one weak and one inessential), one 10-ring and the one 12-ring $(12 d)$, common for both tilings, to construct a unique tiling that has signature $4\left[6^{3}\right]+2\left[4^{2} \cdot 6^{2}\right]+\left[4^{2} \cdot 6^{4} \cdot 10^{2}\right]+\left[4^{8} \cdot 6^{2} \cdot 10^{2} \cdot 12^{2}\right]$ $+\left[4^{12} \cdot 6^{2} \cdot 12^{2}\right]$. The transitivity is 512136 (six kinds of tile as there are two different $\left[6^{3}\right]$ tiles).

The most complicated zeolite net we have examined, that of IM-5 (Baerlocher et al., 2007), has a natural tiling with transitivity 24474119 . In all, we found 281 different tiles in zeolite nets.

In our work, we also use a local program $3 d t$ written by one of us (ODF) that accepts tiling data from TOPOS in the form of coordinates of vertices on a representative of each kind of face and a space group that is used only to generate symmetryrelated faces. $3 d t$ then computes the Delaney-Dress symbol (see e.g. Delgado-Friedrichs et al., 1999) and from that computes the true symmetry (which may be different from that input), transitivity and signature of the tiling, checks to see if it obeys rules $(b)$ and $(c)$, and finds an embedding suitable for illustration (all the figures in this paper were made using $3 d t$ ).

At the time this work was completed, the RCSR database had 1401 distinct topologies (i.e. eliminating alternative embeddings of the same net, and interpenetrating nets) and all have been examined by TOPOS. For 550 of these there was just one proper tiling. ${ }^{1}$ Applying also rules $(b)$ and $(c)$ results in a unique tiling for 1266 nets. For a further 92 , rule $(d)$ results in a unique tiling so we have natural tilings for a total of 1358 nets $(97 \%$ of all). Of the remaining nets, four had multiple tilings and for 39 no tiling was found due to self-entanglement of the net. For seven cases, tiles could be split according to rule (e). Details of the tilings are being incorporated in the RCSR database.

All the 176 recognized zeolite nets (most of these are not in the RCSR) yielded a unique natural tiling, but for four of

\footnotetext{
${ }^{1}$ TOPOS considers only rings and strong rings so does not find the rare cases in which a proper tiling can be constructed using cycles that are not rings as faces. These of course violate rule $(b)$ automatically.
} 
those rule (e) could be applied. A full analysis of zeolite tiles and tilings will be published elsewhere. A review (DelgadoFriedrichs et al., 2007) described some 3-periodic nets of special interest in crystal design. 26 of the 28 uninodal nets listed there have unique proper tilings, as do 19 of the 30 binodal nets and all have unique tilings using rules $(a)-(c)$. We remark that most of the nets of greatest interest in crystal chemistry (including all zeolite nets) have embeddings in which there are no inter-vertex distances shorter than edge lengths (see Delgado-Friedrichs et al., 2005) and it is mainly with these that we are concerned. But it should be recognized that these are just a miniscule part of the infinite universe of nets, most of which probably will not admit tilings at all.

Work at ASU was supported by grants from the US National Science Foundation (grant No. DMR 0451443) and by the donors of the American Chemical Society Petroleum Research Fund.

\section{References}

Baburin, I., Blatov, V. A., Carlucci, L., Ciani, G. \& Proserpio, D. M. (2005). J. Solid State Chem. 178 2452-2474.

Baerlocher, Ch., Gramm, F., Massüger, L., McCusker, L. B., He, Z., Hovmöller, S. \& Zou, X. (2007). Science, 315 1113-1116.

Berger, F., Flamm, C., Gleiss, P. M., Leydold, J. \& Stadler, P. F. (2004). J. Chem. Inf. Comput. Sci. 44, 324-334.

Blatov, V. A. (2006). IUCr CompComm Newsletter, 7, 4-38.

Blatov, V. A. (2007). Acta Cryst. A63, 329-343.

Blatov, V. A., Carlucci, L., Ciani, G. \& Proserpio, D. M. (2004). CrystEngComm, 6, 377-395.

Blatov, V. A., Ilyushin, G. D., Blatova, O. A., Anurova, N. A., Ivanov-Schits, A. K. \& Dem'yanets, L. N. (2006). Acta Cryst. B62, 1010-1018.

Bonneau, C., Delgado-Friedrichs, O., O'Keeffe, M. \& Yaghi, O. M. (2004). Acta Cryst. A60, 517-520.

Carlucci, L., Ciani, G. \& Proserpio, D. M. (2003a). Coord. Chem. Rev. 246, 247-289.
Carlucci, L., Ciani, G. \& Proserpio, D. M. (2003b). CrystEngComm, 5, 269-279.

Delgado-Friedrichs, O., Dress, A. W. M., Huson, D. H., Klinowsky, J. \& Mackay, A. L. (1999). Nature (London), 400, 644-647.

Delgado-Friedrichs, O., Foster, M. D., O'Keeffe, M., Proserpio, D. M., Treacy, M. M. J. \& Yaghi, O. M. (2005). J. Solid State Chem. 178, 2533-2554.

Delgado-Friedrichs, O. \& Huson, D. H. (2000). Discr. Comput. Geom. 24, 279-292.

Delgado-Friedrichs, O. \& O'Keeffe, M. (2003). Acta Cryst. A59, 351-360.

Delgado-Friedrichs, O. \& O'Keeffe, M. (2005a). Acta Cryst. A61, 358-362.

Delgado-Friedrichs, O. \& O'Keeffe, M. (2005b). J. Solid State Chem. 178, 2480-2485.

Delgado-Friedrichs, O. \& O'Keeffe, M. (2006). Acta Cryst. A62, 228-229.

Delgado-Friedrichs, O. \& O'Keeffe, M. (2007). Acta Cryst. A63, 344-347.

Delgado Friedrichs, O., O'Keeffe, M. \& Yaghi, O. M. (2003a). Acta Cryst. A59, 22-27.

Delgado Friedrichs, O., O'Keeffe, M. \& Yaghi, O. M. (2003b). Acta Cryst. A59, 515-525.

Delgado-Friedrichs, O., O'Keeffe, M. \& Yaghi, O. M. (2006). Acta Cryst. A62, 350-355.

Delgado-Friedrichs, O., O'Keeffe, M. \& Yaghi, O. M. (2007). Phys. Chem. Chem. Phys. 9, 1035-1043.

Goetzke, R. \& Klein, H.-J. (1991). J. Non-Cryst. Solids, 127, 215-220.

Hyde, S. T., Delgado-Friedrichs, O., Ramsden, S. J. \& Robins, V. (2006). Solid State Sci. 8, 740-752.

O'Keeffe, M. (1991). Z. Kristallogr. 196, 21-37.

O'Keeffe, M. \& Hyde, B. G. (1996).Crystal Structures I: Patterns and Symmetry. Washington, DC: Mineralogical Society of America.

Schoen, A. H. (1970). Infinite Periodic Minimal Surfaces without Selfintersections. Technical Note D-5541. Washington: National Aeronautics and Space Administration.

Treacy, M. M. J., Randall, K. H., Rao, S., Perry, J. A. \& Chadi, D. A. (1997). Z. Kristallogr. 212, 768-791.

Treacy, M. M. J., Rivin, I., Balkovsky, L., Randall, K. H. \& Foster, M. D. (2004). Microporous Mesoporous Mater. 74, 121-132.

Wells, A. F. (1977) Three-Dimensional Nets and Polyhedra. Wiley: New York.

Wells, A. F. (1979). Further Studies of Three-Dimensional Nets. Am. Crystallogr. Assoc. Monograph No. 8. 